\title{
EMG based input and control system for lower limb prostheses
}

von Rosenberg, Harald

Dennerlein, Florian

Fraunhofer IPA

DE 70569 Stuttgart, Nobelstraße 12

\section{Introduction}

Our goal is the development of a voluntary control input system for lower limb prostheses. For this purpose we use electromyography (EMG) with skin surface electrodes which is a common method for non-invasive measurement of electrical potential during muscle contraction which has not yet been applied successfully to lower limb prostheses due to the difficult signal conditions within the prosthesis socket. Our system will be integrated in the prosthesis socket, making the control system comfortable to wear and easy to use as no electrodes or other parts must be glued to the skin or manually repositioned and placed every day the prosthesis is worn. The control system is designed to allow the user to control a special function within an active powered prosthesis system, e.g. lifting the foot during walking or climbing stairs which are elementary tasks, performed by the subconscious mind of an non-amputee.

The control signal is derived by using remaining muscles of the residual limb. The active prosthesis function can be triggered either by a distinct stimulation of the muscle which is observed or by the subconscious muscle stimulation remaining after the amputation.

Our development offers a potential for further applications like computer input devices, security and safety application, sports and gaming or consumer device control and is not limited to medical applications.

\section{System concept}

Our solution uses an EMG sensor array with direct skin contact as shown in Figure 1. The sensor array consists of up to 16 distinct electrodes generating an eight channel bipolar signal stream. The processing of the sensor values within a pattern recognition system can be performed as shown in Figure 2 where each channel is subject to its own pattern recognition system. A subsequent decision logic will then combine the individual results to form the user's voluntary control signal (WKS). Besides the limited computational and energy resources on active prostheses this would require a more demanding learning and parametrization of the pattern recognition system as the sensor array can move on the skin which will influence the signal which each electrode captures.

To solve this issue we propose the generation of a "virtual EMG" signal that is superior to each single channel representing the best EMG muscle reading, even if the skin and surface is moving below the sensor array. Signal artifacts and disturbances are reduced in this virtual EMG system and the subsequent signal processing based on a set of pattern-recognition algorithms is reduced in complexity as the input signal is minimized to a single sensor signal leading to less demand on computational power.
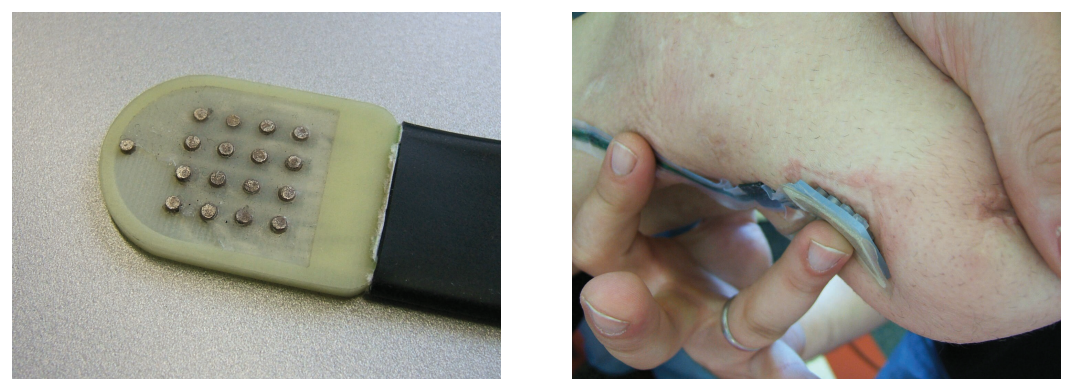

Figure 1: The EMG sensor array is placed onto the skin of the residual limb. 


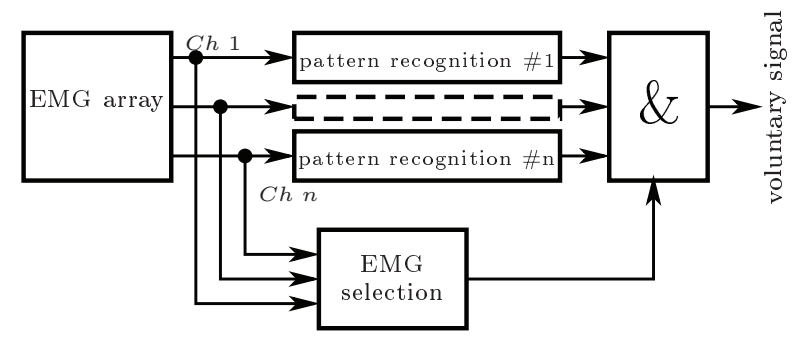

Figure 2: Architecture for merging individual channels to a final result using a signal fusion after individual pattern recognition blocks.

An example of the "virtual EMG" is shown in Figure 3 and the Figure 4 summarizes the processing of the virtual EMG signal within the cocntrol system.
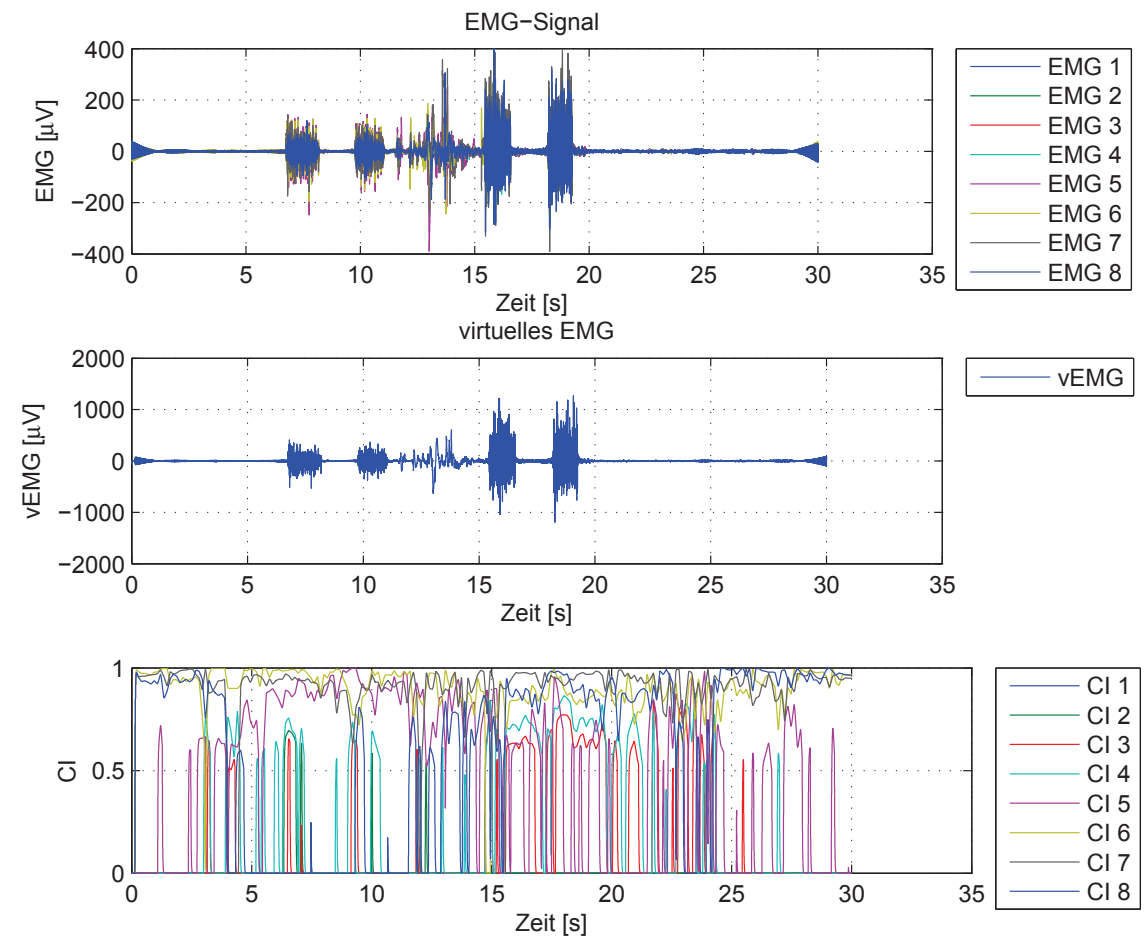

Figure 3: Processing of the virtual EMG signal: on top the 8 input EMG channels are plotted. In the time interval $t=12-15 s$ strong disturbances are visible. In the middle the calculated virtual EMG is presented being cleared by this artifacts. At bottom the calculated factors are shown which indicate the grade $[0 \ldots 1]$ of the EMG channel quality. For example channel 6 has a high belief during the whole measurement whereas other channels are often suppressed.

Due to the EMG signal's severe dependency on the current motion, which the specific muscle is involved in, the motion of the patient must be captured to allow proper detection of the user's voluntary control signal. We use a combination of low-cost inertial navigation system and two pressure sensors on the foot of the patient as well as the pressure information from the EMG sensor array to detect the current motion type and state of the patient.

The main processing system which is shown in Figure 5 provides a motion detection logic, which alters the EMG pattern recognition system, switching between different feature sets and classifiers according to the most probable motion state. This motion classification is also performed in real-time and covers four typical situations, 


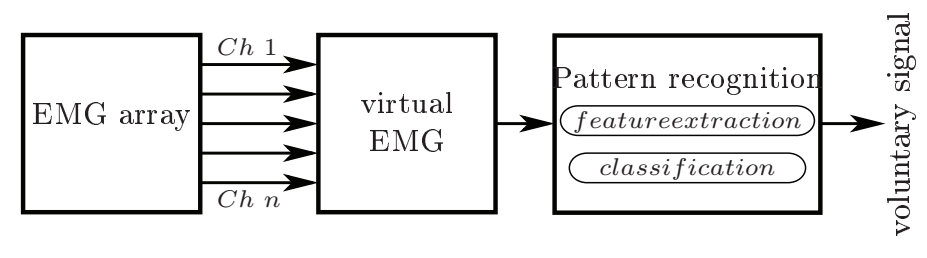

Figure 4: Proposed architecture for merging of individual EMG signal channels.

in which a voluntary prosthesis control signal is of high importance: sitting, standing, walking and climbing an obstacle or stairs.

The EMG accuracy and robustness, with respect to safety aspects and miss-classification of the EMG input signal, can be augmented depending on the usage scenario by dividing the gait cycle into different sections where a control signal is allowed or not.

A set of features is calculated from the virtual EMG signal forming a feature vector. We use time domain and frequency domain features with focus on small processing windows due to real time requirements. A big part of the feature set is derived by discrete wavelet decomposition of the virtual EMG signal. The highly dimensional feature vector passes through a Principal Component Analysis (PCA) where its dimensionality is shrunken. We investigated the PCA with 3, 6 and 9 most important output features in the feature vector. The prepared feature vector is then sent to the classifier. We found that Support Vector Machines (SVM) are superior to other classifiers (e.g. Linear Discriminant Analysis (LDA)).

The learning and parametrization process of the system is reduced due to the strict modularization and interfaces in the signal processing system, as we do not consider the processing as a black box system. User independent parameters (e.g. motion estimation) will not require personal training. Therefore we assume that our trials performed on a single person having a lower limb amputation can be generalized.

The sensor array for capturing the EMG signal is equipped with a pressure sensor array of the same size and number of sensing elements which aids in the calculation of the virtual EMG signal, as electrodes with too low or too high skin contact pressure are reliably detected. An Inertial measurement unit (IMU) is used for orientation measurement and detection of dynamics and identification of type of motion. Additional pressure sensors are located in the prosthesis foot. The overall measurement setup during the experiments is shown in Figure 6. During the measurements the proband held a switch in his hand which he pressed when he triggered the voluntary signal in his limb muscle. This signal was synchronized with the EMG readings and served as a reference signal for the signal analysis and the training of the pattern recognition system.

\section{Results}

Figure 7 and Figure 8 show two exemplary results: on top is the classification result $[0 ; 1]$ of the individual SVMs which are trained for their corresponding motion state. The results are plotted with small offset for better distinction and visibility. Below is the result of the motion state detection shown represented by the motion state's probability $[0 \ldots 1]$. The motion state estimation is followed in the figure by the estimated gait cycle phase (heel strike is starting of gait cycle, next heel strike of the same foot finishes the gait cycle) which serves as an additional source for making the classifier more robust. At bottom the final voluntary signal is presented which could be used as control signal in the active prosthesis system.

The two figures show that the SVM classifier will fail if it is applied to a motion state it was not designed for. Even in a quite simple motion state like standing (Figure 7) heavy false classification occurs. Based on the motion state probability the results of the individual SVMs are combined leading to an accurate voluntary signal detection which can be clearly seen in both figures at the bottom

Compared to the approach in Figure 2 and regarding only one motion state, the virtual EMG offers the advantage that the training data and parameter set of the classifier is more general than training the classifier for every single EMG channel. With respect to the required motion state depending classification the signal processing complexity would otherwise become too big for embedded processing units which fit in an active prosthesis. 


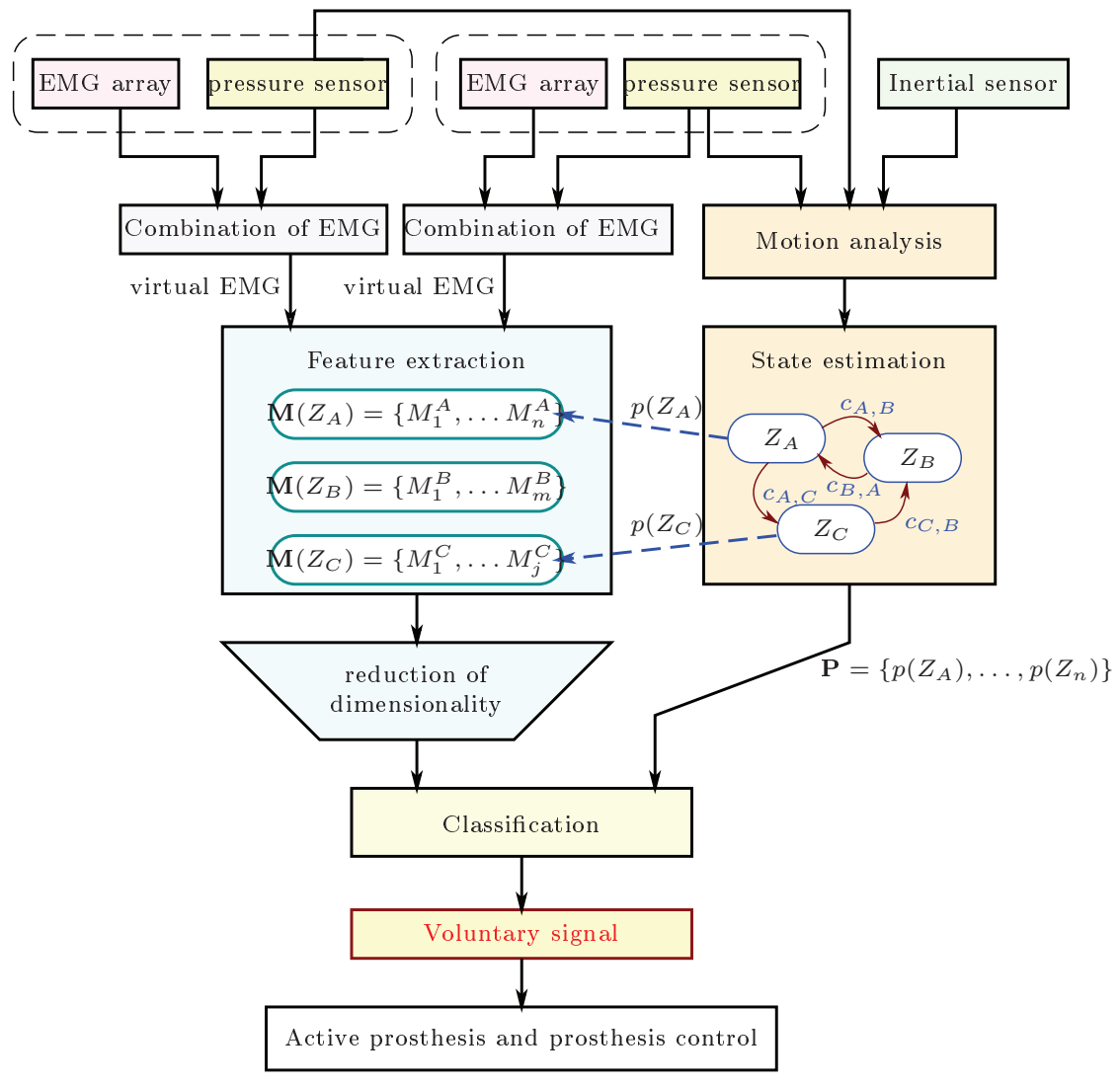

Figure 5: Architecture of the complete pattern recognition and signal processing system.
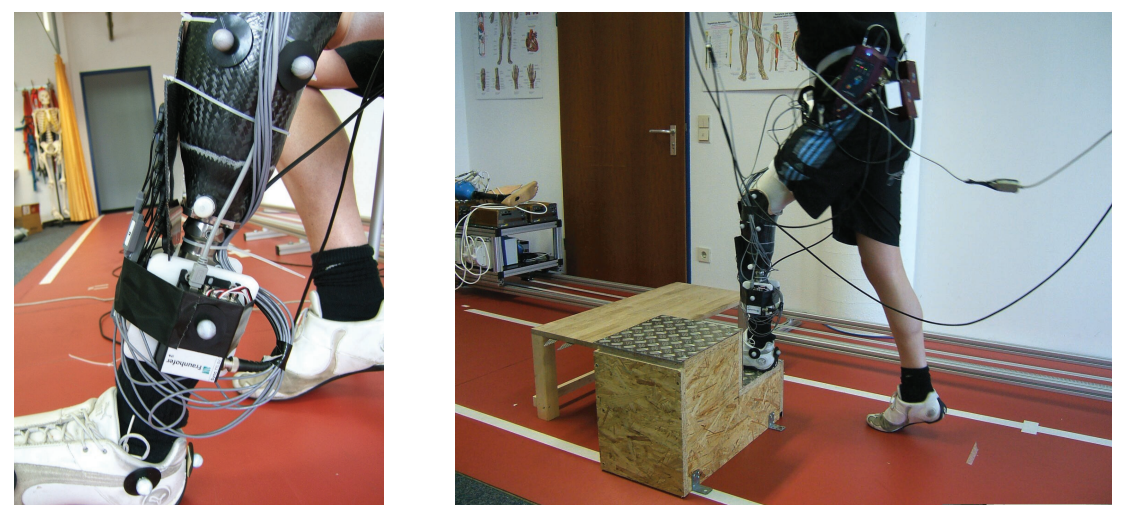

Figure 6: left: Measurement setup - the EMG sensor array is placed inside the prosthesis socket. The inertial measurement unit is located on the outside. Additional infrared markers are attached for comparison with a motion capture system.

right: Trials were performed in the gait laboratory at Fraunhofer IPA showing a stair climbing experiment. 

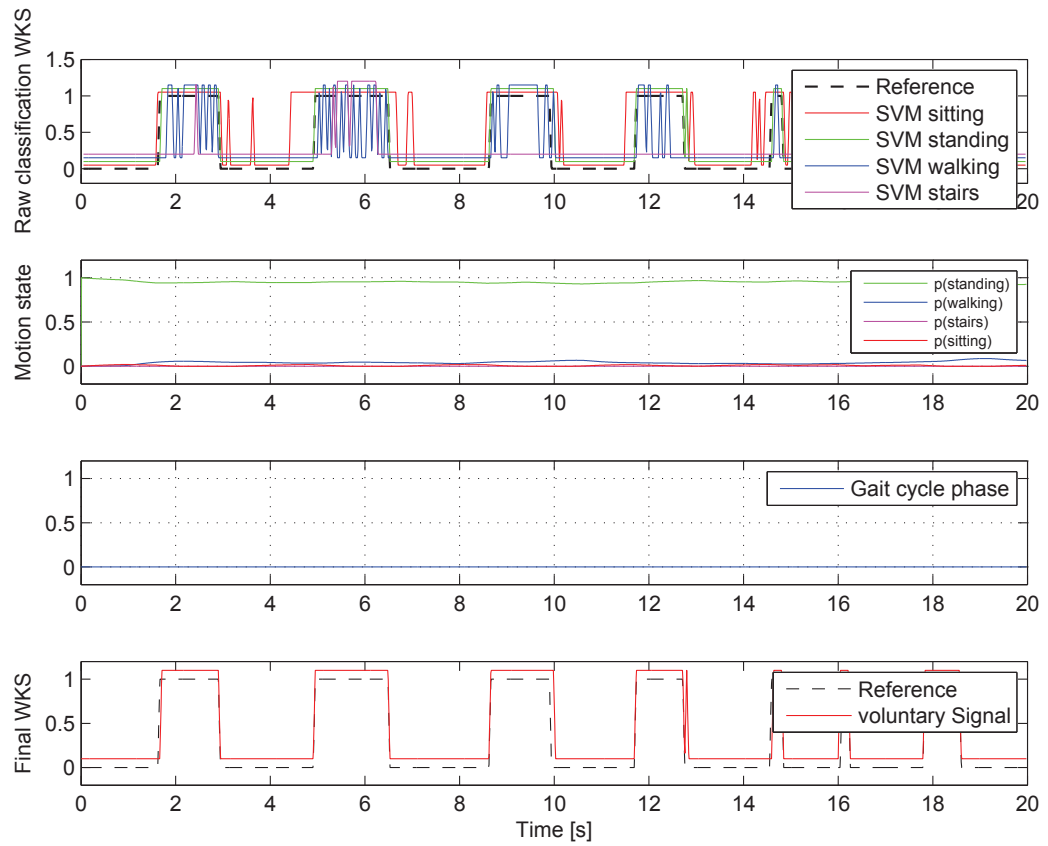

Figure 7: Results for a measurement while standing: The single SVM classifiers detect the voluntary signal but only the "SVM standing" achieves perfect accuracy which is kept in the final voluntary control signal.
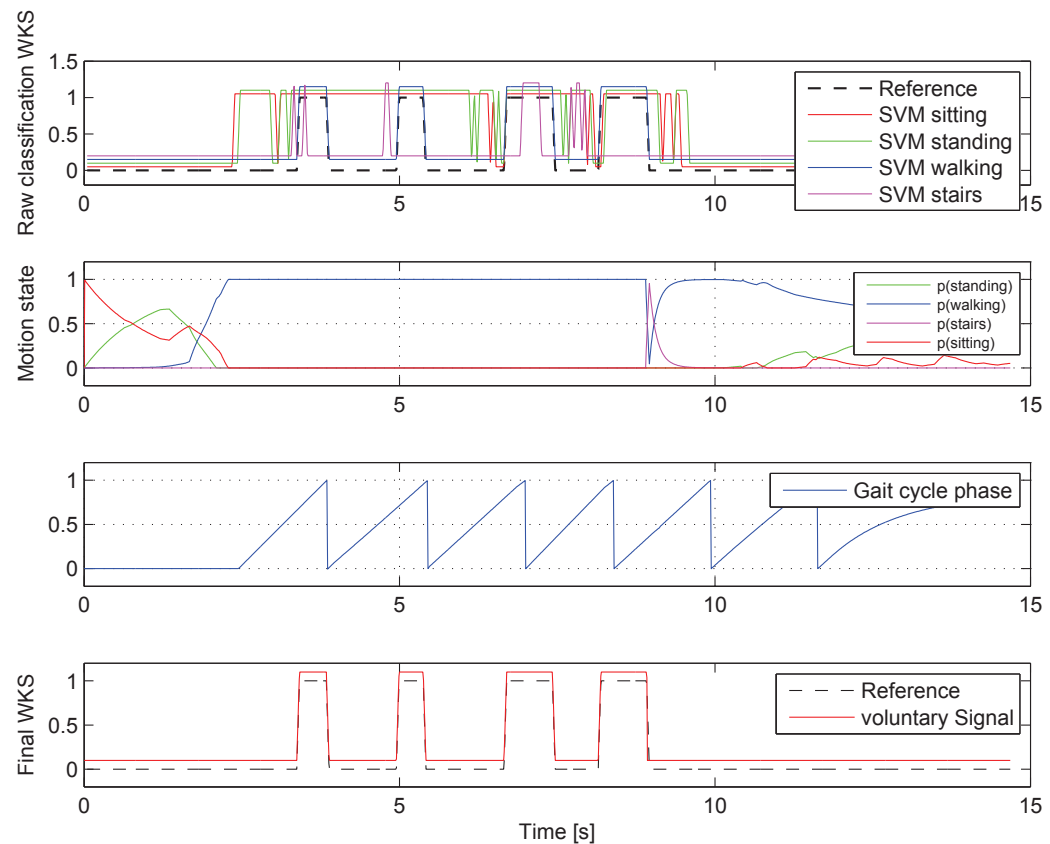

Figure 8: Results for a walking experiment: The SVMs for motion state "sitting" and "standing" fail and only the SVM "stairs" has some correct classifications. Only the SVM "walking" achieves exact classification result compared to the reference signal. In this experiment the final voluntary signal also achieves perfect accuracy. 